\title{
Diagnostic imaging of suspected scaphoid fractures: A literature review
}

\author{
Mohammed K.M. Ali ${ }^{1}$, Mohamed F.M. Elamin ${ }^{2}$, Ahmed M. Mohamed ${ }^{3}$ \\ 1. Registrar Trauma and Orthopedics, Royal Derby Hospital, United Kingdom. 2. Basildon and Thurrock University Hospital, \\ United Kingdom. 3. Specialty Trainee in Clinical radiology, Health Education North East, United Kingdom \\ Correspondence: Mohammed K.M. Ali. Address: Registrar Trauma and Orthopedics, Royal Derby Hospital, United \\ Kingdom. Email: Mohammedkhider84@hotmail.com
}

Received: December 29, 2015

Accepted: January 27, $2016 \quad$ Online Published: February 16, 2016

DOI : $10.5430 /$ ijdi.v3n1p76

URL: http://dx.doi.org/10.5430/ijdi.v3n1p76

\section{Abstract}

The scaphoid plays a pivotal role in the movement of the wrist carpal complex. Scaphoid fractures can be difficult to assess clinically with relatively innocuous symptoms. Avascular necrosis, mal-union or non-union can complicate scaphoid fractures. Early diagnosis and treatment improves outcomes and reduces complication rates. Imaging plays a vital role in the diagnosis and management of such fractures, reducing significant long-term disability. We will discuss the different diagnostic modalities, techniques and protocols for diagnosing Scaphoid fractures.

\section{Keywords}

Suspected scaphoid fracture, Imaging modalities

\section{I ntroduction}

The Scaphoid plays a pivotal role in the wrist carpal complex. It preserves normal alignment of the carpal bones creating normal wrist motion and as such, complications may lead to significant long-term disability ${ }^{[1]}$. Scaphoid fractures commonly happen in youth and they represent about $4 \%$ of all fractures ${ }^{[2]}$ (see Figure 1 ).

Imaging plays a vital role in diagnosing such fractures, as it can be difficult to assess clinically with relatively innocuous symptoms.

Early diagnosis is very important, as any kinematic abnormalities will produce significant interfragmentary motion that will affect the normal fracture healing. The ensuing spontaneous collapse of the two scaphoid fragments will dorsally angulate the wrist producing a "humpback" deformity ${ }^{[3]}$.

Avascular necrosis, mal-union or non-union can complicate scaphoid fractures ${ }^{[4-6]}$. The major blood supply to the scaphoid bone enters through the posterior ridge at the middle of the bone and the volar tubercle at the far end of the scaphoid. The dorsal branch of the radial artery contributes to the majority of the blood supply of the scaphoid. A distinct volar arterial branch to the scaphoid enters the tubercle and contributes to $20 \%-30 \%$ of the blood supply, mostly to the distal portion. The proximal pole of the scaphoid depends solely on intramedullary blood flow. The rare retrograde pattern of the scaphoid's blood supply makes it more susceptible to non-union and proximal pole avascular necrosis ${ }^{[7,8]}$. 
Figure 1. Scaphoid fracture on plain radiographs

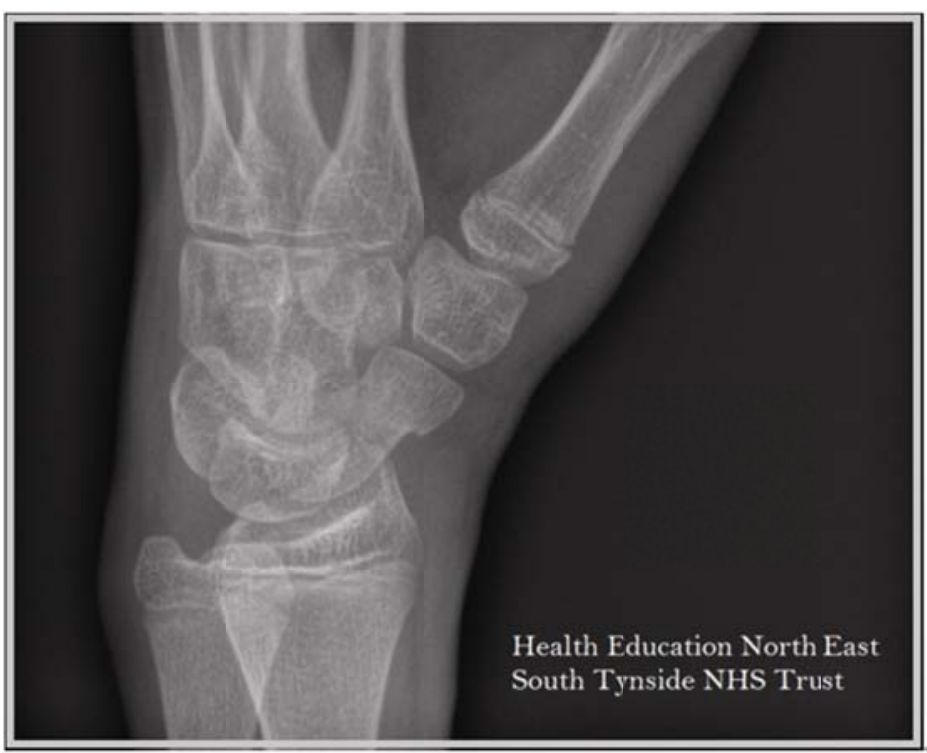

Early diagnosis and treatment improves the outcome and reduces the complication rates. Some authors have argued that subtle fractures, which are not visible on the initial radiographs, are likely to heal spontaneously without immobilisation. However, this view is not widely shared, as Scaphoid fractures are very difficult to put out of action, since almost any

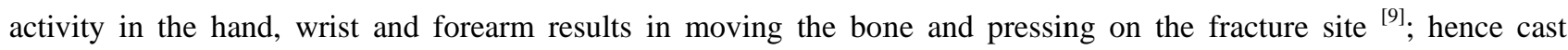
immobilisation is still the treatment of choice. In the contrary, early remobilisation of patients in whom a fracture has been excluded, will significantly reduce disruption to daily life.

\section{What are the diagnostic options?}

A wide range of diagnostic modalities, techniques and protocols are used worldwide. These include repeat plain radiographs, Bone Scintigraphy, Magnetic Resonance Imaging (MRI) and Computed Tomography (CT) imaging (see Figures 2, 3).

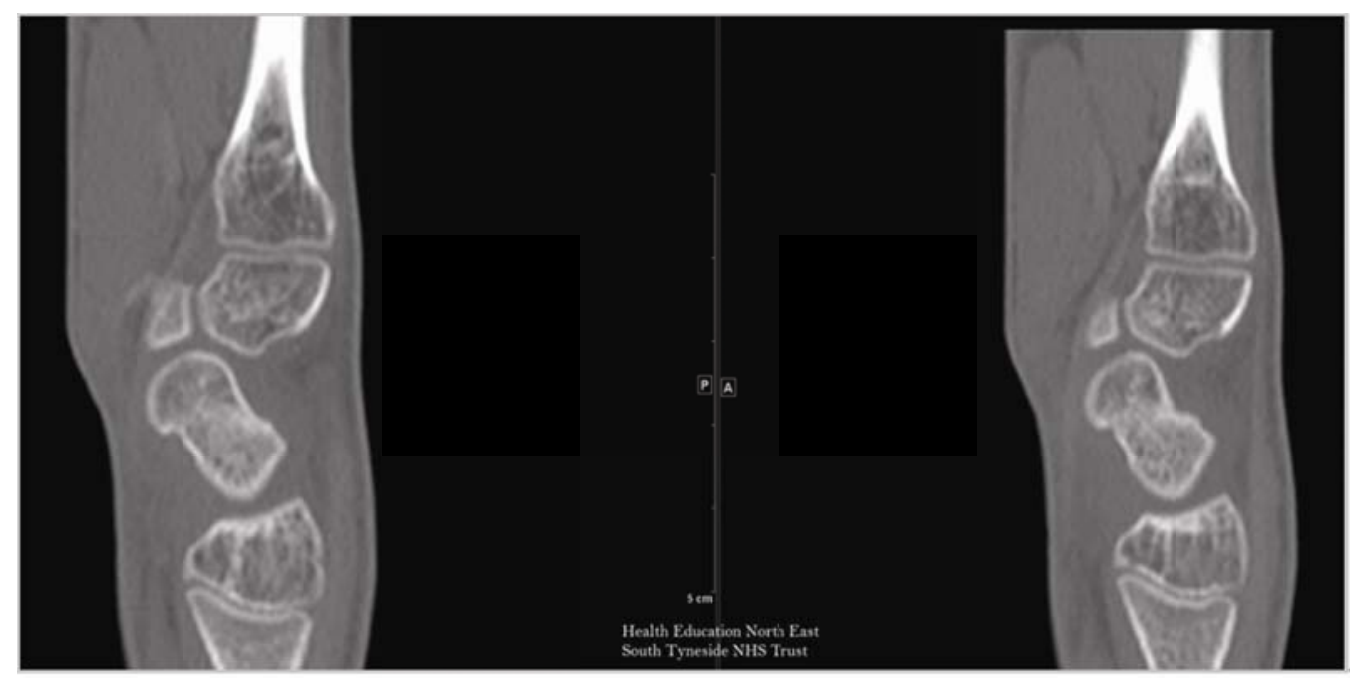

Figure 2. Scaphoid fracture on CT scan 
The majority of centres adopt the approach of repeating plain radiographs after a suitable time interval along with re-examination. However, a subset of patients with a negative repeat radiograph remains symptomatic. This sets the stage for a second-line diagnostic modality, which will reduce un-necessary in-cast time.

Most studies have agreed that following a normal initial radiograph; further evaluation should be carried out in all symptomatic patients. In 2005, Low et al. ${ }^{[10]}$ conducted a study to see whether repeat radiographs are reliable tests. In this study, four expert observers independently reviewed fifty sets of initial and follow-up radiographs looking for Scaphoid fracture. MRI was used as a gold standard to check the sensitivity, specificity, positive and negative predictive values of the observers' assessment of the follow-up radiographs. Add to that, they performed a reliability variance analysis among the observers. The study concluded that; plain radiographs had very low diagnostic value and reliability when performed to examine suspected occult scaphoid fractures. The study also concluded that follow-up radiographs, performed after normal initial radiographs, have no great diagnostic value.

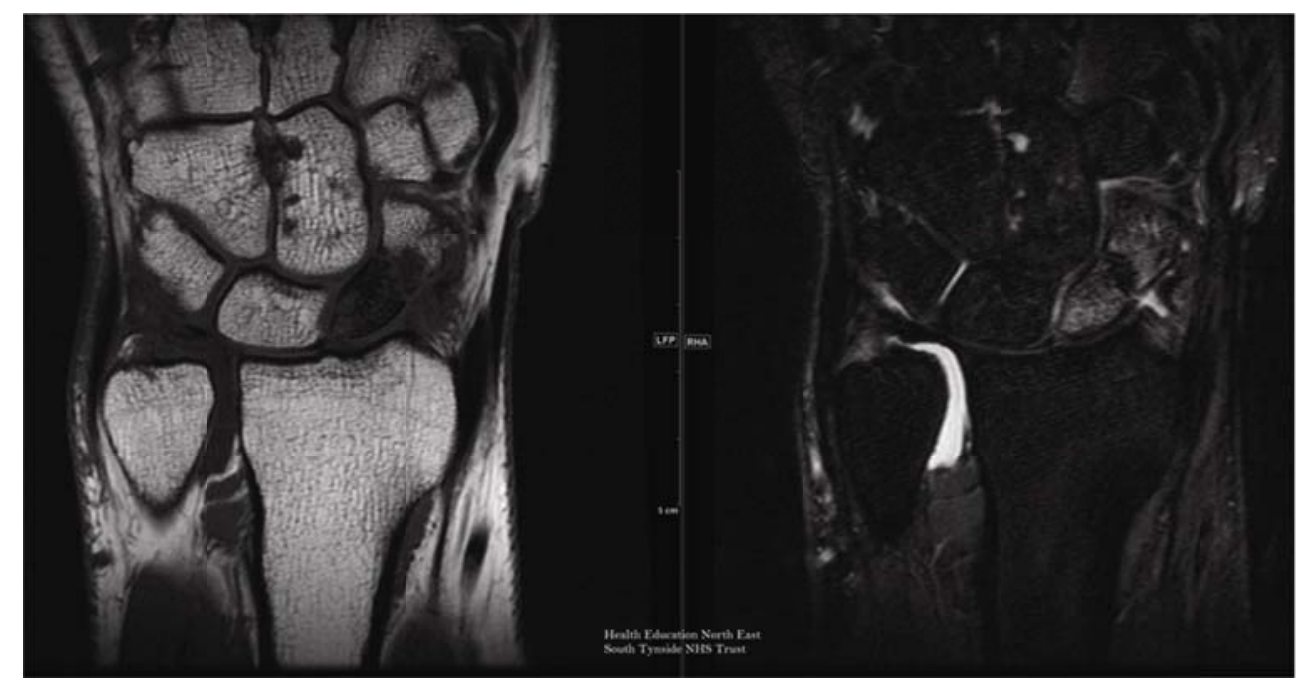

Figure 3. Shows scaphoid fracture on MRI scan

A large pool of evidence, however, reports a wide spectrum of sensitivity figures and recommendations on the ideal modality, for example, a study by Beeres et al. in $2008{ }^{[11]}$ compared early MRI to Bone Scintigraphy. They reviewed a hundred patients with a suspected scaphoid fracture but with non-conclusive radiographs using MRI and bone Scintigraphy. MRI was performed within one day of injury, and bone scintigraphy three to five days after injury. Their reference standard for a true radiologically-occult scaphoid fracture was either a diagnosis of fracture on both MRI and bone scintigraphy, or, in the case of discrepancy, clinical and/or radiological evidence of a fracture. It was concluded that early, short-sequence MRI was not better than bone scintigraphy for the diagnosis of a suspected Scaphoid fracture and recommended that bone scintigraphy is a highly sensitive and specific test for the diagnosis of suspected Scaphoid fracture. Zong et al. in $2010{ }^{[12]}$ conducted a systematic review and meta-analysis for twenty-six studies in order to assess and compare the diagnostic abilities of bone scintigraphy, MRI, and CT for diagnosing occult scaphoid fractures. They concluded that Bone scintigraphy and MRI have equally higher sensitivity and higher diagnostic value than CT scan when ruling out scaphoid fracture. However, MRI is more specific and considered the best for confirming scaphoid fracture.

There is a strong financial argument for this, as some studies have shown that a limited early MRI may cost the same or not significantly more than the cost of cast, repeat consultation with an Orthopaedic specialist and repeat radiographs; excluding the financial implications of interval immobilisation to the patient ${ }^{[13,14]}$. Karl et al. in 2015 created a decision analysis model to assess three diagnostic protocols for patients with occult scaphoid fracture. The first protocol was immobilising the cast empirically and repeating radiographs with an orthopaedic follow-up at two weeks post-injury. The second protocol involved doing immediate CT scanning and the third protocol was performing an immediate MRI. 
Prevalence of occult scaphoid fracture, sensitivity and specificity of CT and MRI, and risks and outcomes of a missed fracture were derived from published literature. Costs of imaging, lost worker productivity and surgical costs of non-union surgery were estimated based on the literature. They reported that advanced imaging had lower costs and health outcomes compared to those of empiric cast immobilization. MRI was slightly more cost-effective than CT on the basis of the mean published diagnostic performance, but was highly sensitive to test performance characteristics ${ }^{[15]}$.

There is tight comparison between the sensitivities of all three. Second line modalities made it acceptable for centres to choose whatever suits their preference and local resources. A large international survey of 105 hospitals in 6 continents has shown that only $21.9 \%$ had fixed written protocols for imaging of the scaphoid. $72 \%$ of the hospitals would do repeat radiographs after 10-14 days before moving to another modality. 5.7\% of hospitals relied solely on repeat radiographs and clinical examination for managing scaphoid injuries. Overall, MRI was the most commonly used second line in 57 centres, 47 for CT and 35 for bone scintigraphy. In Europe, CT was the most favourable. Hospitals in North America were in favour of MRI; this is likely to be related to the American College of Radiology (ACR) recommendations ${ }^{[16]}$. The ACR also deemed Bone Scintigraphy inappropriate as it carries an unjustifiably high radiation dose in the presence of cross sectional modalities.

\section{Which imaging modality is better?}

Early plain radiographs are clearly the least sensitive. This is markedly improved in the delayed radiographs due bone resorption and remodulative widening of the fracture line. Some studies have concluded that if a fracture is not visible on first day; it will be difficult to visualize in delayed plain radiographs ${ }^{[17]}$. The limitations of radiographs are exaggerated further by the wide interobserver variability during reporting. This affects both accuracy and reproducibility (consistency) of reports ${ }^{[18,19]}$. This was shown to improve with experience, which implies that at least the repeat radiographs should be reported or reviewed by a senior reporting observer, especially if a second line investigation is to be considered. A recent study by Edlund et al. investigated the sensitivity of cone beam computed tomography (CBCT), a low dose technique recently made available for extremity examinations, in detecting scaphoid fractures. MRI was used as gold standard for scaphoid fractures. The study concluded that CBCT is a superior alternative to radiography, entailing more accurate diagnoses of carpal region fractures, and thereby requiring fewer follow-up MRI examinations. However, CBCT cannot be used to exclude scaphoid fractures, since MRI identified additional occult scaphoid fractures ${ }^{[20]}$.

A slightly larger number of authors have favoured MRI for the imaging of occult scaphoid injuries ${ }^{[21,22]}$. Our own observation of the literature has concluded that the sensitivity of CT is becoming comparable to MRI in the more recent studies. We can logically attribute that to the huge improvement in CT multi-detector technology and multi-planar capabilities. Memarsadhegi et al. in 2006 found MRI and CT to be comparable in overall sensitivity. In this study, Twenty-nine patients had multi-detector CT and MRI within 6 days after injury. CT data were acquired with 0.5-mm collimation. For image review, 0.7-mm-thick multi-planar reformations were performed in sagittal, transverse and coronal planes. The 1.0-T MR examination consisted of coronal and transverse short inversion time inversion-recovery, coronal and transverse T1-weighted spin-echo, and coronal volume-rendered T2- weighted gradient-echo sequences. The MR and CT images were reviewed by two radiologists. A binomial examination was performed to assess the amount of the discrepancies between MR imaging and CT in visualising scaphoid fractures and cortical involvement ( $P$.05). The study determined that CT was superior in depicting cortical involvement, while MRI edged ahead in trabecular fractures ${ }^{[23]}$. This may prove significant as, although MRI is of higher sensitivity across the literature pool, it failed to differentiate between cortical and purely trabecular fractures on a few occasions. Some evidence has shown that incomplete fractures (without cortical involvement) are significantly less likely to develop non-union ${ }^{[24]}$. This concludes that CT is capable of detecting the significant fractures that are likely to need intervention. However, MRI's ability to detect ligamentous and cartilaginous injuries is non debatable ${ }^{[25,26]}$. 
Questions arise; if any immediate intervention will be undertaken or any long-term sequelae in relation to these soft tissue injuries will help sway the preference more in favour of MRI.

Historically, these fractures in children and adolescents have commonly involved the distal pole, requiring neither surgical care nor extended follow-up, however recent studies concluded that scaphoid fracture anatomy in children and adolescents are now similar to the published patterns in adults. While the majority of acute non-displaced fractures heal with conservative treatment, three months of cast immobilization or more may be needed for more proximal injuries. About $30 \%$ of paediatric scaphoid fractures will present late with chronic non-unions ${ }^{[27,28]}$. Some authors prefer immediate immobilisation without further imaging; however, the majority suggest performing an additional diagnostic examination ${ }^{[30-32]}$. MRI is the most favourable examination in order to minimise the dose of ionizing radiation ${ }^{[30]}$, and also suitable for ruling out anatomical variations ${ }^{[33,34]}$

\section{So what should we do?}

Our literature review has concluded a few recommendations:

1) A follow up radiograph and re-examination should be carried out at 10-14 days. An experienced observer should interpret these radiographs.

2) MRI and CT are of comparable sensitivity.

3) The modality of choice depends on cost, availability and reporter's preference, which can differ from one centre to another.

4) MRI has more evidence to support its marginally superior sensitivity. This, combined with the lack of ionising radiation, makes it the modality of choice if readily available.

5) Tailored fast MRI sequence protocols should be used to limit scanning time.

6) Special considerations should be given to young patients, skilled manual workers and athletes. Rapid evaluation by MRI rather than delayed repeat radiographs is advised for this group to aid management and to limit unnecessary immobilisation.

\section{Conflict of interests}

There is no competing interest regarding to this article.

\section{Funding}

No Funding from any organisation has been received.

\section{References}

[1] Berdia S, Wolfe SW. Effects of scaphoid fractures on the biomechanics of the wrist. J Bone Joint Surg Br. 2008; 90(9): 1205-9.

[2] Steven JR, Daan O, Frank JPBs. Current methods of diagnosis and treatment of scaphoid fractures. Int J Emerg Med. 2011 ; 4: 4. http://dx.doi.org/10.1186/1865-1380-4-4

[3] Smith DK, Cooney WP, KN An. The effects of simulated unstable scaphoid fractures on carpal motion. J Hand Surg Am. 1989; 14(2 Pt 1): 283-91. http://dx.doi.org/10.1016/0363-5023(89)90022-1

[4] Kozin SH. Incidence, mechanism, and natural history of scaphoid fractures. Hand Clin. 2001; 17(4): 515-24. PMid:11775464

[5] Peter CA, Thomas HB, Douglas KS. Scaphoid malunion. 1989; 14(4): 679-687. http://dx.doi.org/10.1016/0363-5023(89)90191-3 
[6] Coblenz G, Christopoulos G, Fröhner S. Scaphoid fracture and nonunion: current status of radiological diagnostics. Radiologe. 2006; 46(8): 664-676. http://dx.doi.org/10.1007/s00117-006-1398-z

[7] Gaebler C, McQueen MM. Carpus fractures and dislocations. In: Bucholz RW, Heckman JD, Court-Brown CM, Tornetta P, editors. Fractures in adults. Philadelphia: Lippincott Williams and Wilkins. 2009: 782-828.

[8] Barton NJ. Twenty questions about scaphoid fractures. J Hand Surg Br. 1992; 17(3): 289-310. http://dx.doi.org/10.1016/0266-7681(92)90118-L

[9] Kaneshiro SA, Failla JM, Tashman S. Scaphoid fracture displacement with forearm rotation in a short-arm thumb spica cast. J Hand Surg Am. 1999; 24(5): 984-91. PMid:10509277 http://dx.doi.org/10.1053/jhsu.1999.0984

[10] Low G, Raby N. Can follow-up radiography for acute scaphoid fracture still be considered a valid investigation? Clin Radiol. 2005; 60(10): 1106-10. PMid:16179171 http://dx.doi.org/10.1016/j.crad.2005.07.001

[11] Beeres FJ, Rhemrev SJ, den Hollander P. Early magnetic resonance imaging compared with bone scintigraphy in suspected scaphoid fractures. J Bone Joint Surg Br. 2008; 90(9): 1205-9. http://dx.doi.org/10.1302/0301-620X.90B9.20341

[12] Yin ZG, Zhang JB, Kan SL. Diagnosing suspected scaphoid fractures: a systematic review and meta-analysis. Clin Orthop Relat Res. 2010; 468(3): 723-34. PMid:19756904 http://dx.doi.org/10.1007/s11999-009-1081-6

[13] Theodore AD, Nancy MM, Clyde AH. Cost-Effectiveness of Immediate MR Imaging Versus Traditional Follow-Up for Revealing Radiographically Occult Scaphoid Fractures. AJR. 2001; 177: 1257-1263. PMid:11717059 http://dx.doi.org/10.2214/ajr.177.6.1771257

[14] Brooks S, Cicuttini FM, Lim S. Cost effectiveness of adding magnetic resonance imaging to the usual management of suspected scaphoid fractures. Br J Sports Med. 2005; 39: 75-79. http://dx.doi.org/10.1136/bjsm.2003.007435

[15] Karl JW, Swart E, Strauch RJ. Diagnosis of Occult Scaphoid Fractures: A Cost-Effectiveness Analysis. J Bone Joint Surg Am. 2015; 97(22): 1860-8. http://dx.doi.org/10.2106/JBJS.O.00099

[16] American College of Radiology (ACR), Expert Panel on Musculoskeletal Imaging, ACR Appropriateness Criteria. Acute hand and wrist trauma. Reston, VA. American College of Radiology. 2008: 1-9.

[17] Available from: http://www.bjjprocs.boneandjoint.org.uk/content/94 B/SUPP_XXXVII/477.abstract

[18] Tiel-van Buul MMC, van Beek EJR, Broekhuizen AH. Diagnosing scaphoid fractures: radiographs cannot be used as a gold standard! Injuries. 1992; 23(2): 77-79. http://dx.doi.org/10.1016/0020-1383(92)90035-Q

[19] Dias JJ, Thompson J, Barton NJ. Suspected scaphoid fractures: the value of radiographs. J Bone Joint surg [Br]. 72-B: 98-101.

[20] Edlund R, Skorpil M, Lapidus G. Cone-Beam CT in diagnosis of scaphoid fractures. Skeletal Radiol. 2016; 45(2): 197-204. PMid:26563560 http://dx.doi.org/10.1007/s00256-015-2290-6

[21] Raby N. Magnetic resonance imaging of suspected scaphoid fractures using a low field dedicated extremity MR system. Clin Radiol. 2001; 56: 316-320. http://dx.doi.org/10.1053/crad.2000.0657

[22] Mack MG, Keim S, Balzer JO. Clinical impact of MRI in acute wrist fractures. Eur Radiol. 2003; 13: 612-617. PMid:12594566

[23] Memarsadhegi. Occult Scaphoid Fractures: CT versus MR Imaging. Radiology. 2006; 240(1).

[24] Pao VS, Chang J. Scaphoid nonunion: diagnosis and treatment. Plastic Reconstructive Surgery. 2003; 112: $1666-1676$. PMid:14578801 http://dx.doi.org/10.1097/01.PRS.0000086090.43085.66

[25] Kitsis C1, Taylor M, Chandey J. Imaging the problem scaphoid. Injury. 1998; 29(7): 515-20. http://dx.doi.org/10.1016/S0020-1383(98)00115-6

[26] Hodgson RJ, O’Connor PJ, Grainger AJ. Tendon and ligament imaging. Br J Radiol. 2012; 85(1016): 1157-1172. PMid:22553301 http://dx.doi.org/10.1259/bjr/34786470

[27] Gholson JJ1, Bae DS, Zurakowski D. Scaphoid fractures in children and adolescents: contemporary injury patterns and factors influencing time to union. J Bone Joint Surg Am. 2011; 93(13): 1210-9. PMid:21776574

[28] Ahmed I, Ashton F, Tay WK. The pediatric fracture of the scaphoid in patients aged 13 years and under: an epidemiological study. J Pediatr Orthop. 2014; 34(2): 150-4. PMid:24172664 http://dx.doi.org/10.1097/BPO.0000000000000102

[29] Wilson EB, Beattie TF, Wilkinson AG. Epidemiological review and proposed management of "scaphoid” injury in children. Eur J Emerg Med. 2011; 18(1): 57-61. PMid:20585258 http://dx.doi.org/10.1097/MEJ.0b013e32833c9312

[30] Lögters TT, Linhart W, Schubert D. Diagnostic approach for suspected scaphoid fractures in children. Eur J Trauma Emerg Surg. 2008; 34(2): 131-4. PMid:26815617 http://dx.doi.org/10.1007/s00068-007-7037-1

[31] Weber DM. Scaphoid fractures in childhood. Unfallchirurg. 2011; 114(4): 285-91. PMid:21445650 http://dx.doi.org/10.1007/s00113-011-1963-4 
[32] Foley K, Patel S. Fractures of the scaphoid, capitate and triquetrum in a child: a case report. J Orthop Surg. 2012; 20(1): 103-4.

[33] Bhatti AN, Griffin SJ, Wenham SJ. Deceptive appearance of a normal variant of scaphoid bone in a teenage patient: a diagnostic challenge. Orthop Rev. 2012; 4(1): 33-4.

[34] Fabre O, De Boeck H, Haentjens P. Fractures and nonunions of the carpal scaphoid in children. Acta Orthop Belg. 2001; 67(2): 121-5. PMid:11383289 